Amptek Inc.

Tel: 781-275-2242

Email: sales@amptek.com

www.amptek.com

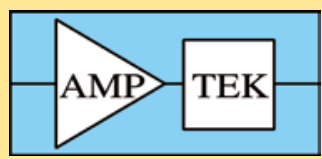

FAST SDD ${ }^{\circledR}$ Detector for EDS (SEM)

APPLICATIONS: $\bullet$ Detectors $\bullet$ Energy-Dispersive $X$-ray Spectrometry (EDS) - Spectrometers (X-ray) - X-ray Analysis Equipment $\bullet$ Scintillators

FEATURES: SDD detectors for EDS-XRF use with SEMs by OEMs. Low energy response of the Amptek FAST SDD ${ }^{\circledR}$ extends down to carbon (C).

http://www.amptek.com/products/eds-sem-applications-with-c2-window/

\title{
Basler Inc.
}

Tel: +1 610-717-5517

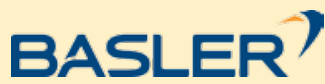

Email: sales.usa@baslerweb.com www.baslerweb.com

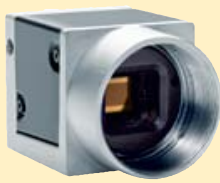

\section{Digital Microscope Cameras}

APPLICATIONS: • Camera/Digital Camera Systems - Cameras, CCD • Cameras, CMOS

FEATURES: Leading digital camera technology (GigE, USB 3.0) and over 25 years of vision technology experience. Basler cameras offer easy integration, compact size, excellent image quality and an outstanding price/performance ratio.

www.baslerweb.com/microscopy

\section{CoolLED Limited}

Tel: +1 800-877-0128

Email: info@coolled.com

www.coolled.com

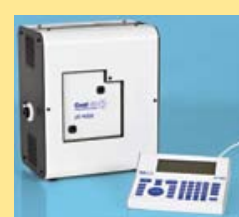

\section{CoolLED PE-Series LED Illumination} Systems

APPLICATIONS: • Fluorescence Microscopy • Illuminators (LOM) • Lamps and Light Sources • LED Illumination (LOM) • LED Lamps

FEATURES: Contact CoolLED's U.S. office to learn more about our wide range of LED illumination systems for fluorescence and Optogenetics.

http://www.coolled.com/products/

\section{Daylight Solutions, Inc.}

Tel: 858-432-7533

Email: mbarre@daylightsolutions.com www.daylightsolutions.com

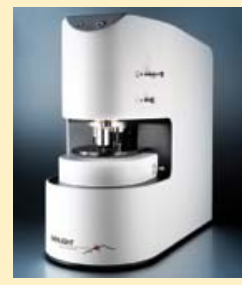

\section{Spero Infrared Microscope}

APPLICATIONS: • FT-IR Microscopy • Infra-Red Microscopes • Spectrometers (light) • Lasers • Raman Spectroscopy/Microscopy

FEATURES: Spero is the world's first laser-based infrared microscopy platform and provides: • High spatial resolution chemical imaging $\bullet$ Rapid data acquisition - Discrete frequency imaging • Uncooled operation

\section{Deben}

Tel: +44 (0) 1359244 870;

+12014105028 (Angstrom Scientific)

Email: paulg@deben.co.uk

www.deben.co.uk

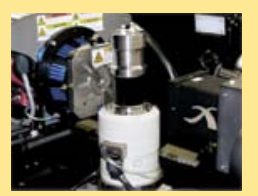

In Situ Testing for Microscopy

APPLICATIONS: Deben manufacture in-situ testing stages as well as innovative accessories for SEM and TEM including SEM detectors, stages, stage automation and Peltier heating \& cooling stages.

FEATURES: - Micro CT tensile scanning stages - Centaurus scintillator CL and back scattered electron (BSE) detectors - STEM detectors for SEM - SEM heating and cooling Peltier stages - Microtest tensile and compression stages

http://deben.co.uk/products/

\section{Diatome U.S.}

Tel: 215-412-8390

Email:sgkcck@aol.com

www.emsdiasum.com

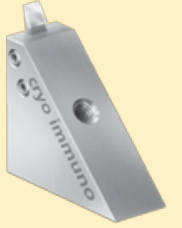

\section{Cryo Immuno}

The first cryo knife with a diamond platform and $35^{\circ}$ cutting angle that leads to a considerable reduction in mechanical stresses and therefore to improved structure preservation in sucrose-protected samples.

\section{Electron Microscopy Sciences}

Tel: 215-412-8400

E-mail: stacie@ems-secure.com www.emsdiasum.com

\section{Electron Microscopy Sciences}

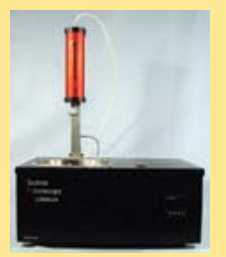

\section{EMS-002 Cryo Workstation}

The EMS-002 Cryo Workstation is a complete ultra rapid freezing system that captures rapid events and labile structures that are not seen in chemically fixed materials.

\section{Evans Analytical Group}

Tel: 408-530-3500

Email: helpdesk@eag.com

www.eag.com $/ \mathrm{mc}$

\section{Material Characterization}

APPLICATIONS: - Microscopy Services - Surface Analysis • Service Laboratories $\bullet$ Microanalysis Services - Failure Analysis

FEATURES: Providing high quality microscopy services including: SEM, S/TEM, EDS, EELS, EBIC, CL, EBSD, FIB imaging and now: Aberration Corrected STEM services using a Hitachi HD-2700. 


\section{Excelitas Technologies E (X-Cite ${ }^{\circledR}$ )}

Tel: +1 800-668-8752

Email:X-Cite@excelitas.com

www.excelitas.com

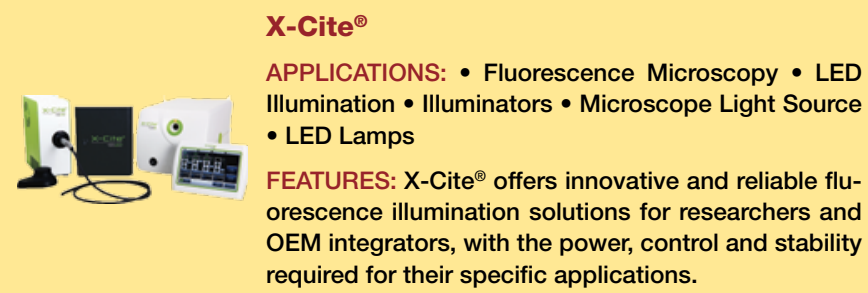

www.excelitas.com/X-Cite

\section{ibss Group, Inc.}

Tel: 415-566-5774

Email: admin@ibssgroup.com

ibssgroup.com

\section{GV10x DS Asher}

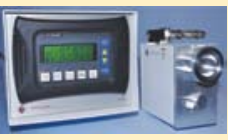

APPLICATIONS: • Contamination Control $\bullet$ Long $\mathrm{mfp}$ Plasma Cleaning - New In-Situ Cleaning Paradigm - FIB/SEM/TEM • SIMS/XPS/Review SEM

FEATURES: Unique plasma source is the engine in GV10x DS Asher that provides high power and low pressure long mfp plasma for cleaning chambers and specimens in-situ.

ibssgroup.com

\section{EXpressLO LLC}

Tel: +1 321-663-3806

Email: info@EXpressLO.com

www.EXpressLO.com

EXpressLOTM EX Situ

Lift Out System

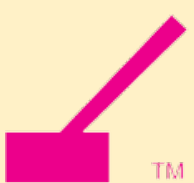

EXpressLO LLC

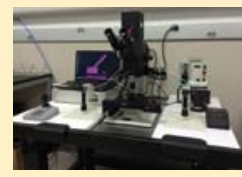

APPLICATIONS: - FIB Accessories - FIB Lift Out - TEM Grids - TEM Specimen Preparation - APT Specimen Preparation

FEATURES: EXpressLO'T allows fast and easy ex situ lift out - no carbon support film needed. No expensive FIB time required. Simple manipulation for routine backside milling reduces curtaining.

www.EXpressLO.com

\section{JPK Instruments AG}

Tel: +49 30-726243-500

Email: Bagordo@jpk.com www.jpk.com

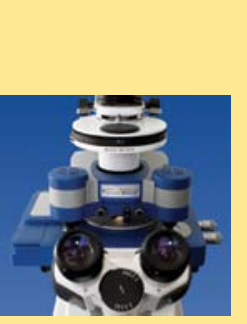

\section{NanoWizard ${ }^{\circledR}$ ULTRA Speed A AFM}

APPLICATIONS: JPK is a leading manufacturer of nano-analytical instruments-atomic force microscope (AFM) and optical tweezers systems-for life sciences and soft matter applications.

FEATURES: • Fast scanning $>100 \mathrm{~Hz}$ line rate in air and liquid - Atomic resolution in closed-loop mode - Easy-to-use QITM mode for quantitative material property mapping - Integration with optical microscopy by tip-scanning design and DirectOverlay ${ }^{\mathrm{TM}}$ - Force measurements from single molecules to living cells

http://usa.jpk.com/nanowizard-r-ultra-speed-a-overview.830.us.html

\section{LaVision BioTec}

Tel: +49 (0) 5219151390

\section{Hitachi High Technologies America, Inc.}

Tel: 800-548-9001

Email: kyoko.ramirez@hitachi-hta.com www.hitachi-hta.com

Electron, Ion, and Probe Microscopy

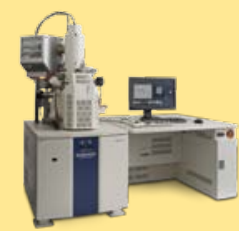

APPLICATIONS: • Scanning Electron Microscopes (SEMs) • Transmission Electron Microscopes (TEMs) - Atomic Force Microscopes (AFMs) - Focused Ion Beam Systems • Ion Beam Milling

FEATURES: Advanced solutions for materials science, biological research, and industrial manufacturing. Standard \& VP-SEM, TEM \& STEM, FIB, tabletop SEM, AFM, and microanalysis sample preparation systems.

www.hitachi-hta.com/products/electron-ion-and-probe-microscopy
Email: pingel@lavisionbiotec.com

www.lavisonbiotec.com

\section{TriM Scope II 2-Photon Microscope}

APPLICATIONS: LaVision develops and manufactures advanced microscopy solutions for life sciences. Trim Scope II - modular multi-photon/confocal microscopy platform; UltraMicroscope II - light sheet microscope platform.

FEATURES: Fast intravital imaging with Cloud and resonant Scanner - FRAP, photoactivation, photostimulation, uncaging and line imaging with Cloud Scanner • SHG and THG imaging with OPO and Ti:Sa laser - Integrated FLIM imaging with LaVision BioTec's FLIM x16 TCSPC detector - Ultrafast 64 beam imaging with up to 100 frames/s @ 2560x2160 pixel

http://www.lavisionbiotec.com/trim-scope-overview.html 


\section{Leica Microsystems}

Tel: 800-248-0123

Fax: 846-405-0164

Email: yourimage@leica-microsystems.com

www.leica-microsystems.com

\section{Leica EM CPD Critical Point Dryer}

APPLICATIONS: • Life Sciences • Materials Science - Critical Point Drying - SEM Sample Preparation - High-quality Samples

FEATURES: Dries delicate biological or industrial samples in a fully automated, controlled process to preserve surface structure for subsequent treatment and analysis.

http://www.leica-microsystems.com/products/em-sample-prep/biologicalspecimens/room-temperature-techniques/drying/details/product/leicaem-cpd300/

\section{Leica EM VCT100 Vacuum Cryo Transfer System

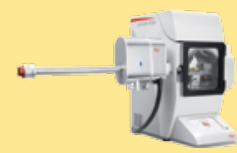 \\ APPLICATIONS: - Life Sciences - Mounts to Any Vacuum-operated SEM, FIB, SIMS, AFM, or XPS Chamber $\bullet$ Contamination-free Cryo Transfer \\ FEATURES: Samples are transferred in a well-defined environment, e.g., protective gas or high vacuum from a preparation instrument to analysis unit: can be} kept at low-temperature for cryo- techniques.

http://www.leica-microsystems.com/products/em-sample-prep/biologicalspecimens/low-temperature-techniques/vacuum-transfer/details/product/ leica-em-vct100/

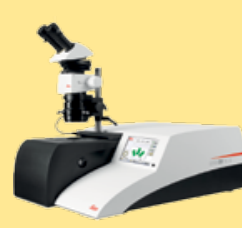

Leica EM TIC 3X Ion Beam Slope Cutter

APPLICATIONS: • Materials Science - Triple Ion Beam Cutter • Cross-sections for SEM, Microstructure Analysis, and AFM • Sample Preparation • Milling at High Rates

FEATURES: For high-quality cross sections of materials, this instrument features three individually controlled ion beams that can cut up to three deep, broad samples in one process.

http://www.leica-microsystems.com/products/em-sample-prep/industrialmaterials/solid-state-technology/details/product/leica-em-tic-3x/

\section{Leica EM ACE Series of Coaters}

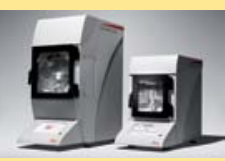

APPLICATIONS: • High vacuum coating for FE-SEM and TEM - Low vacuum coating - Sputter Coating and Carbon Thread $\bullet$ Sample Preparation

FEATURES: The latest advances in automated low vacuum and high vacuum coating systems - one touch coating with a small footprint.

http://www.leica-microsystems.com/products/em-sample-prep/biologicalspecimens/room-temperature-techniques/coating/

\section{Leica EM UC7 Ultramicrotome}

APPLICATIONS: - Life Sciences - Sample Prepara-

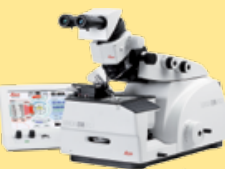
tion for TEM, SEM, AFM, and LM • Room Temperature and Cryo Sectioning $\bullet$ Materials Science $\bullet$ Semi- and Ultra-thin Sections

FEATURES: Provides easy preparation of semi- and ultra-thin sections as well as perfect, smooth surfaces of biological and industrial samples for TEM, SEM, AFM and LM examination.

http://www.leica-microsystems.com/products/em-sample-prep/biologicalspecimens/low-temperature-techniques/ultramicrotomy/details/product/ leica-em-uc7-1/

\section{Linkam Scientific Instruments}

Tel: +44 (0) 1737363476

Email: Duncanstacey@linkam.co.uk

www.linkam.co.uk

\section{THMS600 Heating/Cooling stage}

APPLICATIONS: The most versatile heating and cooling stage available. Precise temperature control. Ideal for use with light microscopy, FT-IR, UV, Raman, $X$-ray, SAXS and Synchrotron.

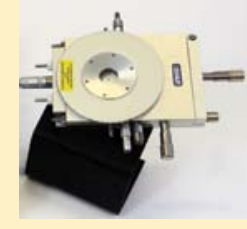

FEATURES: $-196^{\circ} \mathrm{C}$ to $600^{\circ} \mathrm{C}$ temperature range with liquid nitrogen $\bullet$ Heating rate to $150^{\circ} \mathrm{C} / \mathrm{min}$; cooling rate $100^{\circ} \mathrm{C} / \mathrm{min} \bullet 0.1^{\circ} \mathrm{C}$ accuracy and stability with no overshoot - Options for pressure, vacuum, electrical measurements $\bullet$ Mounts to enable vertical operation in IR or X-ray spectrometers

http://www.linkam.co.uk/thms600-features/

\section{CMS196 Cryo CLEM stage}

APPLICATIONS: The Linkam Cryo-CLEM stage allows vitrified samples to be imaged in brightfield and fluorescence without contamination. Grids can be mapped to provide correlative information.

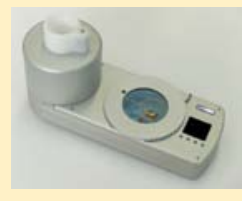

FEATURES: - Self-contained cryo correlative system keeping the sample vitrified at constant $-196^{\circ} \mathrm{C}$ - High precision encoded motorized XY with automated mapping - Short start up time with high longterm stability and low drift - Self-aligning magnetic sample cassette system - Integrated condenser optics for transmitted light - brightfield, phase contrast and fluorescence observation

http://www.linkam.co.uk/clem

\section{Max Levy Autograph}

Tel: 800-798-3675

Email: tom.cinque@ii-vi.com

www.maxlevy.com

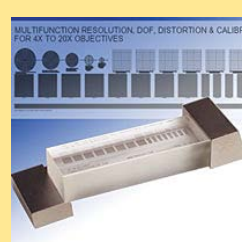

\section{Microscope Metrology Calibration Tools}

APPLICATIONS: • Calibration Standards • Resolution Standards - Rulers - Reference Materials/Reference Standards $\bullet$ Accessories

FEATURES: MLA produces an array of image qualification products to evaluate any microscope or vision system. Components include reticles, resolution, distortion, MTF, and gray scale targets.

http://www.maxlevy.com/category/vision-tools.html

\section{McCrone Microscopes A MCCRONE \& Accessories}

Tel: 630-887-7100

Email: lbolin@mccrone.com

http://www.mccrone.com

\section{NeoScope II Benchtop SEM}

APPLICATIONS: - Benchtop Electron Microscopes - Camera/Digital Camera Systems - Digital Microscope • Freeze Drying Equipment $\bullet$ Heating Stages

FEATURES: Widest magnification range 10X-60,000X - No special sample prep - EDS for elemental analysis - Images in under 3 minutes - Intuitive touchscreen interface

http://www.benchtopsem.com/ 


\section{Microtome Service Company}

Tel: 315-451-1404

Email: mitch@MicrotomeServiceCo.com

http://www.microtomeserviceco.com

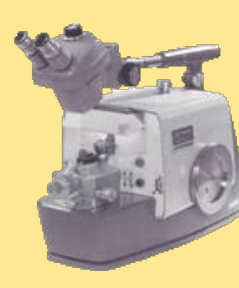

Microtome Sales, Service, and Parts

APPLICATIONS: • Microtomes and Ultramicrotomes - Microtome Repair - Knifemakers - Service and Repair • Fabricate \& modify lab equipment

FEATURES: Sales, service of Sorvall, DuPont and RMC Microtomes and GKM Knife Makers. Microtome \& Lab accessories fabrication and modifications to meet your specific needs.

http://microtomeserviceco.com/index.php?route=information/ information\&information_id=7

\section{NanoMEGAS USA}

Tel: 480-389-6816

Email: robert@appfive.com

www.nanomegasusa.com

Topspin Experiment Framework for (Transmission) Electron Microscopy

APPLICATIONS: • Transmission Electron Microscopes (TEM) • Software for Microscopy • Electron Backscatter Diffraction • Energy-Dispersive X-Ray Spectrometry (EDS) • Scanning Electron Microscopy (SEM)

FEATURES: Novel (S)TEM experiments enabled by synchronized beam scanning, beam precession and advanced analysis - Strain, Orientation, and Phase Mapping • Nanocrystal Characterization • Enhanced EDX/EELS Spectroscopy • in-situ microscopy

www.nanomegasusa.com

\section{Oregon Physics}

Tel: +1 503-601-0041

\section{DREGON}

Email: info@oregon-physics.com

www.oregon-physics.com

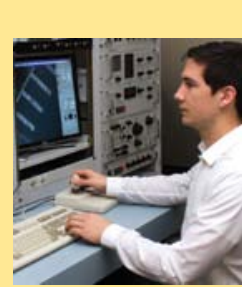

Plasma FIB Service Laboratory

APPLICATIONS: • Ion Beam Milling • Microscopy Services • Plasma FIB Service Laboratory • Sample Preparation - Service Laboratories

FEATURES: Save days or weeks of research time for samples $50 \mu \mathrm{m}$ to $1 \mathrm{~mm}$. Large area cross sections; micromachining slits and apertures; 3D FIB tomography; sample preparation.

www.oregon-physics.com

\section{Pfeiffer Vacuum}

\section{PFEIFFER VACUUM}

Tel: 800-248-8254

Email: Jgiambartolomei@pfeiffer-vacuum.com

www.pfeiffer-vacuum.com

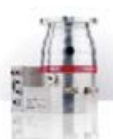

HiPace turbopumps

APPLICATIONS: • Vacuum Equipment • Mass Spectrometers/Residual Gas Analyzers • Gauges, vacuum - Leak Detection

FEATURES: HiPace ${ }^{\circledR}$ turbopumps offer the clearest image utilizing hybrid or magnetic bearing systems resulting in low vibrations, low magnetic stray field and long-term stability and reliability.

http://www.pfeiffer-vacuum.com/products/turbopumps/container.action

\section{Protochips}

Tel: 919-341-2612

Email: megan@protochips.com www.protochips.com

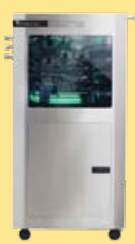

Atmosphere Environmental Gas Cell

APPLICATIONS: • Catalysis • Fuel Cells

FEATURES: Capability to precisely control gas environment at pressures up to 1 ATM and temperatures up to $1000{ }^{\circ} \mathrm{C} \cdot$ SmartTemp ${ }^{\text {TM }}$ closed-loop temperature control for constant known temperature with any gas species or pressure • Fully EDS compatible

http://www.protochips.com/products/atmosphere-gas-environmental-cell. html

\section{Rigaku Corporation}

Tel: +1-512-225-1796

(P) Rigalkw

Email: michael.nelson@rigaku.com www.Rigaku.com

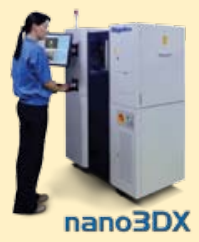

\section{Rigaku nano3DX}

APPLICATIONS: • X-ray Microscope • Computed Tomography

FEATURES: Nano3DX is a X-ray microscope (XRM) with the ability to measure relatively large samples at high resolution using a high powered rotating anode $X$-ray source and a CCD imager.

http://www.rigaku.com/products/xrm/nano3dx

\section{SPI Supplies}

Tel: 610-436-5400

Email: sales@2spi.com www.2spi.com
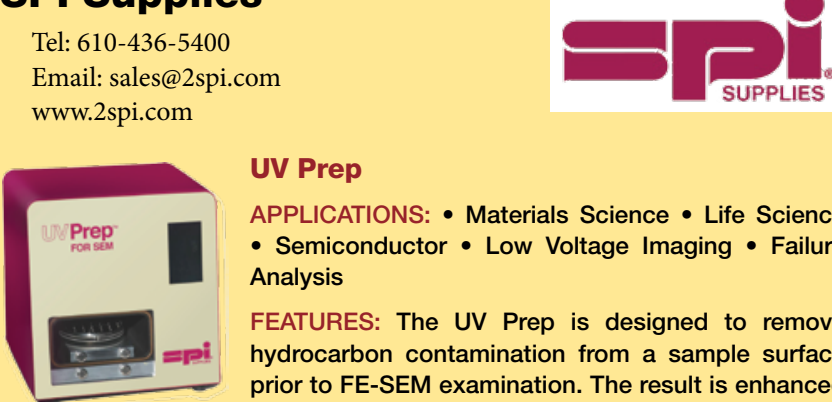

UV Prep

APPLICATIONS: • Materials Science • Life Science - Semiconductor - Low Voltage Imaging • Failure Analysis

FEATURES: The UV Prep is designed to remove hydrocarbon contamination from a sample surface prior to FE-SEM examination. The result is enhanced imaging and resolution at low accelerating voltages.

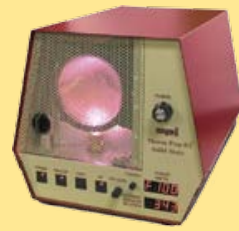

Plasma Prep ${ }^{\text {TM }}$ III

APPLICATIONS: • Materials Science • Life Science - Semiconductor - Asbestos • Failure Analysis

FEATURES: Solid state RF generator capable of 1 to $100 \mathrm{~W}$ operations; Low Temperature asher/ etcher; small footprint; 4" diameter $\times$ 6" depth Pyrex or Quartz Chamber; Optional Process Controller; Optional system for cleaning TEM Specimen Holders.

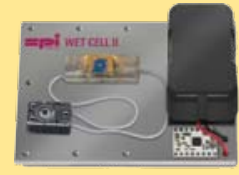

Wet Cell II

APPLICATIONS: • Materials Science • Life Science - Microfluidic Analytical Techniques • Self-contained Interface • Vacuum Compatible

FEATURES: The next generation device for the examination of liquids in SEM/EDS and TOF-SIMS instruments. The self-contained high vacuum compatible device enables the analyst to characterize a fluid in its natural state. 


\section{Sutter Instrument}

Tel: 415-883-0128

Email: alex@sutter.com

www.sutter.com

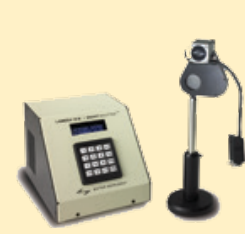

\section{SmartShutter ${ }^{\mathrm{TM}}$}

APPLICATIONS: Shuttering, Fluorescence Microscopy, Life Sciences, Imaging Hardware

FEATURES: • Life tested to 100 million cycles $\bullet$ Complete opening time $8 \mathrm{msec}$ from trigger $\bullet$ Select partial opening function for neutral density capability - "Soft" mode provides minimum vibration - Stand alone, or use with Sutter filter wheel

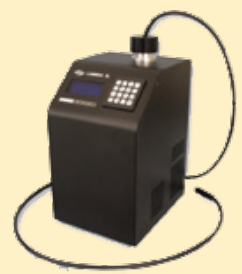

Lambda XL

APPLICATIONS: Microscope Illumination, Fluorescence Microscopy, Life Sciences, Imaging Hardware, OEM Illumination

FEATURES: • 10,000 hour expected lifetime for bulb - No high-voltage pulse $\bullet$ Highly stable output (less than $\pm 1 \%$ fluctuations) - Built-in driver for Sutter filterwheel and SmartShutter $\bullet$ Low light loss through bulb life

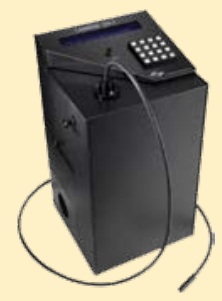

\section{Lambda DG-4 / DG-5 PLUS}

APPLICATIONS: Fluorescence Microscopy, Ratiometric Imaging, Wavelength Switching, Imaging Hardware, Life Sciences

FEATURES: - $0.5 \mathrm{msec}$ switching between filters - Integrated light source and wavelength switching excitation system $\bullet$ Holds four or five interference filters and one neutral density filter $\bullet$ Light guide output provides uniform illumination - Direct computer control via parallel or serial interface

\section{Lambda 10-3}

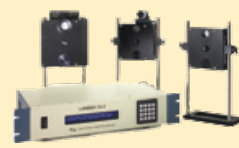

APPLICATIONS: Fluorescence Microscopy, Ratiometric Imaging, Wavelength Switching, Imaging Hardware, Life Sciences

FEATURES: - As fast as $40 \mathrm{msec}$ between adjacent filters (10 position wheel) - As fast as $31 \mathrm{msec}$ between adjacent filters ( 4 position wheel) $\bullet$ Can control up to 3 wheels and 2 shutters, or 2 wheels and 3 shutters - Compatible with SmartShutter ${ }^{\mathrm{TM}}$ stepper-motor controlled shutter - Controls a variety of $12,25,32$, and $50 \mathrm{~mm}$ filterwheels

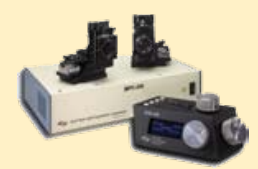

\section{MPC-385 / MPC-325}

APPLICATIONS: Life Sciences, Micromanipulation, Electrophysiology

FEATURES: - Single controller and ROE will run 2 stepper motor drive manipulators - User-friendly interface: single button access to all major functions - Accelerated mode for fast, manual manipulator movement - Definable 4 th axis for coaxial pipette movement - Ultra-low drift, ultra-smooth movement.

\section{SOM-Simple Moving Microscope}

APPLICATIONS: • Fluorescence Microscopy $\bullet$ In vivo microscopy • In vitro microscope • Life sciences - Photostimulation

FEATURES: The SOM is a small, simple microscope designed to allow a single experimental setup to be used for both in vivo and in vitro experimentation.
Ted Pella, Inc.

Tel: 800-237-3526

Email: sales@tedpella.com www.tedpella.com

High Resolution FE-SEM Sputter Coaters APPLICATIONS: Life Sciences, Materials Science, Semiconductors, SEM

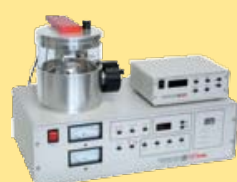

FEATURES: - Grain-free, ultra-thin uniform and conform coating - Wide choice of operating parameters to accommodate all sample types - Purpose designed with optimized vacuum pumping system - Includes Rotary-Planetary-Tilting stage and high resolution thickness controller $\bullet$ Easy to operate with fast cycle times

www.tedpella.com/cressington.htm

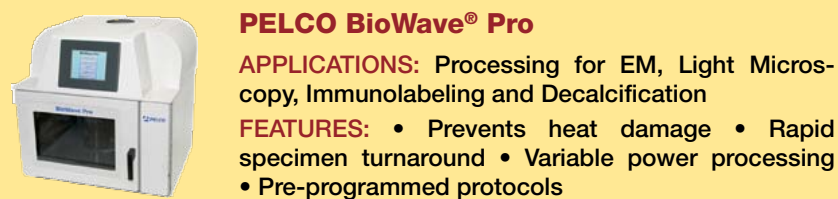
www.tedpella.com/BioWave.htm

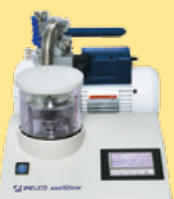

PELCO easiGlow ${ }^{\mathrm{TM}}$

APPLICATIONS: Life Sciences, Materials Science, TEM, Tomography

FEATURES: - Precise and easy vacuum settings - Short cycle times - Consistent results - Intuitive touch screen for control and display - Supports hydrophilic/hydrophobic and negative/positive modes

www.tedpella.com/easiGlow.htm

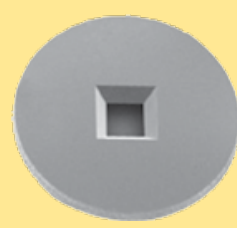

PELCO ${ }^{\circledR}$ Silicon Nitride Support Films and TEM Supplies

APPLICATIONS: TEM, STEM, Thin Film Research, Life Sciences, Materials Science

FEATURES: - Debris-free and resilient films - Available membrane thickness of $8,15,35,50$ and $200 \mathrm{~nm}$ - $3 \mathrm{~mm}$ diameter frame fits perfectly in TEM holders - EasyGrip ${ }^{\mathrm{TM}}$ edges for improved handling

www.tedpella.com/TEM-supplies.htm

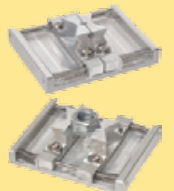

PELCO ${ }^{\circledR}$ Modular SEM Sample Holders and Q Pin Stubs for Correlative Microscopy APPLICATIONS: SEM, FE-SEM, FIB, FIB/SEM, CLEM FEATURES: • Stage adapters for all major SEM brands - Large selection of effective and practical sample holders $\bullet$ Correlative microscopy square sample holders www.tedpella.com/SEM-supplies.htm

\section{Thermo Fisher Scientific \\ ThermoFisher S C I E N T I F I C}

Tel: 1 800-532-4752

Email: info.microanalysis.us@thermofisher.com www.thermoscientific.com/spectroscopy

\section{NORAN System 7 X-ray Microanalysis} System

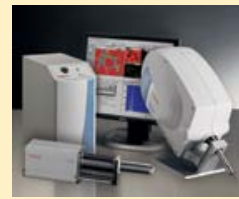

APPLICATIONS: • Energy-Dispersive X-ray Spectroscopy (EDS) - Wavelength-Dispersive X-ray Spectroscopy (WDS) - Electron Backscatter Diffraction (EBSD) • Raman Spectroscopy/Microscopy • FT-IR Spectroscopy/Microscopy

FEATURES: Specializing in intuitive, easy to use microspectroscopy that eliminates operator bias for consistent results. We are the world leader in serving science. When results count, count on us.

www.thermoscientific.com/microanalysis 


\section{Microscopy Product Vendors}

\section{Companies and their Products}

\section{XEI Scientific, Inc.}

Tel: 650-369-0133

Email: sales@evactron.com

www.Evactron.com

\section{Evactron ${ }^{\circledR} 25 Z$ De-Contaminator}

APPLICATIONS: SEM, FIB, and vacuum chamber remote plasma cleaner; carbon removal from vacuum chamber.

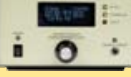

FEATURES: Uses air to make oxygen radicals - Preset cleaning recipes - Available computer interface - Simple operation - Table top controller - Fast Zephyr mode cleaning at $<20$ mTorr

http://evactron.com/25zephyr

\section{Evactron ${ }^{\circledR} 45 Z$ and $40 Z$ De-Contaminators} APPLICATIONS: SEM, FIB, and vacuum chamber remote plasma cleaners; carbon removal from vacuum chamber.

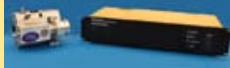

FEATURES: Use air • Preset cleaning recipes • Available computer interfaces - Simple operation - Rack mounted controllers - Fast Zephyr mode cleaning at $<20$ mTorr

http://evactron.com/evactron45; http://evactron.com/evactron40

\section{Evactron ${ }^{\circledR}$ SoftClean ${ }^{\mathrm{TM}}$ Chamber}

APPLICATIONS: Desktop plasma cleaning of specimens; uses one PRS for SEM and specimen cleaning; cleaning without sputter etching

FEATURES: 3 ports for TEM stage holders - Top opening chamber lid for SEM samples - RF shield built into lid $\bullet$ Includes vacuum cutoff valve $\bullet$ Uses air for cleaning gas

http://evactron.com/softclean

\section{Evactron ${ }^{\circledR}$ Plasma Radical Sources}

APPLICATIONS: Make oxygen radicals from air or oxygen gas; make hydrogen radicals from $\mathrm{H} 2$; plasma light sources.

FEATURES: A variety of size and appearance options - Built-in pressure measurement - Built-in gas flow control $\bullet$ Compact size $\bullet$ Easy mounting

http://www.evactron.com/images/files/prs-v.pdf; http://www.evactron.com/images/files/2012PRSu.pdf; http://www.evactron.com/images/files/prs-p.pdf

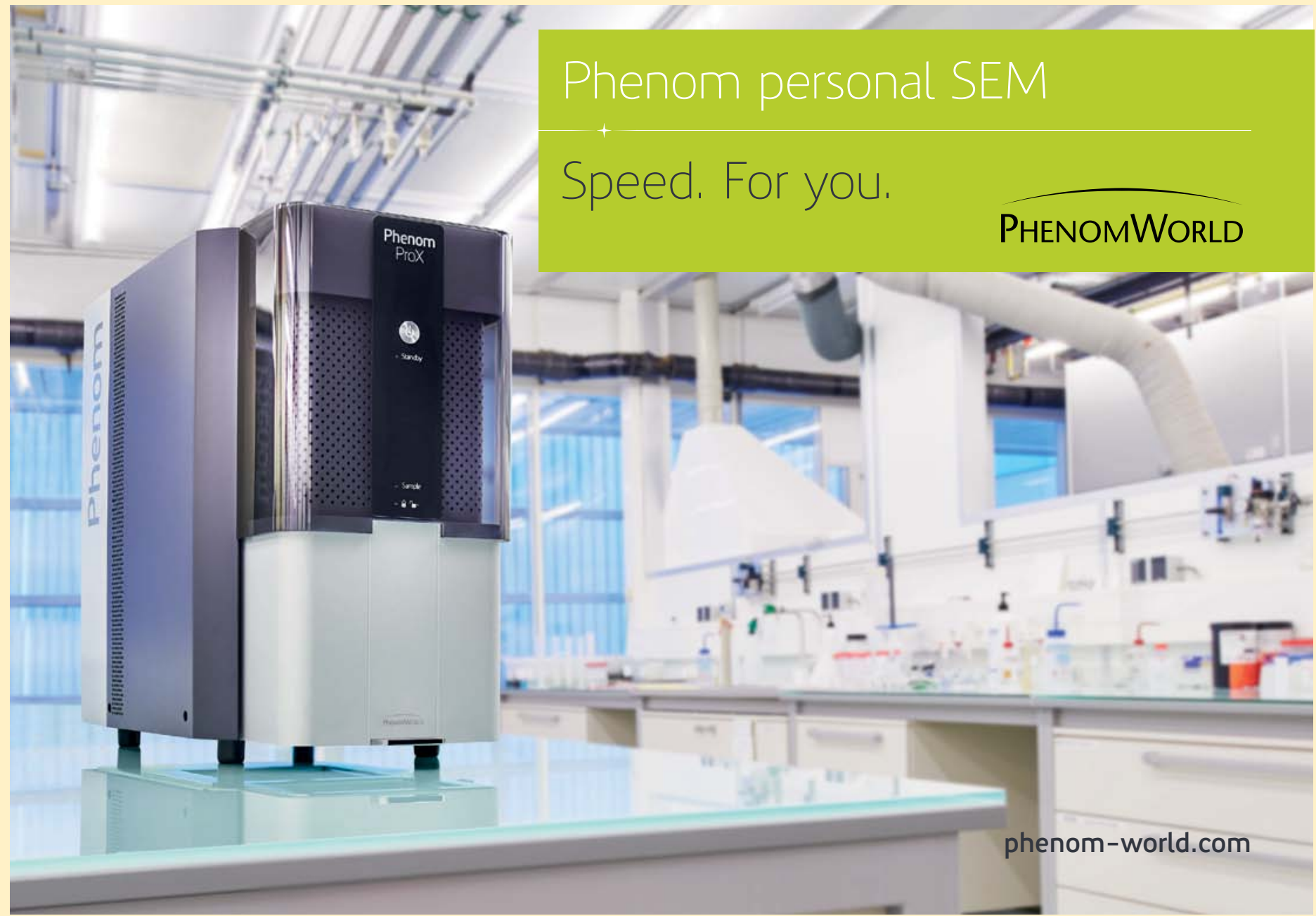




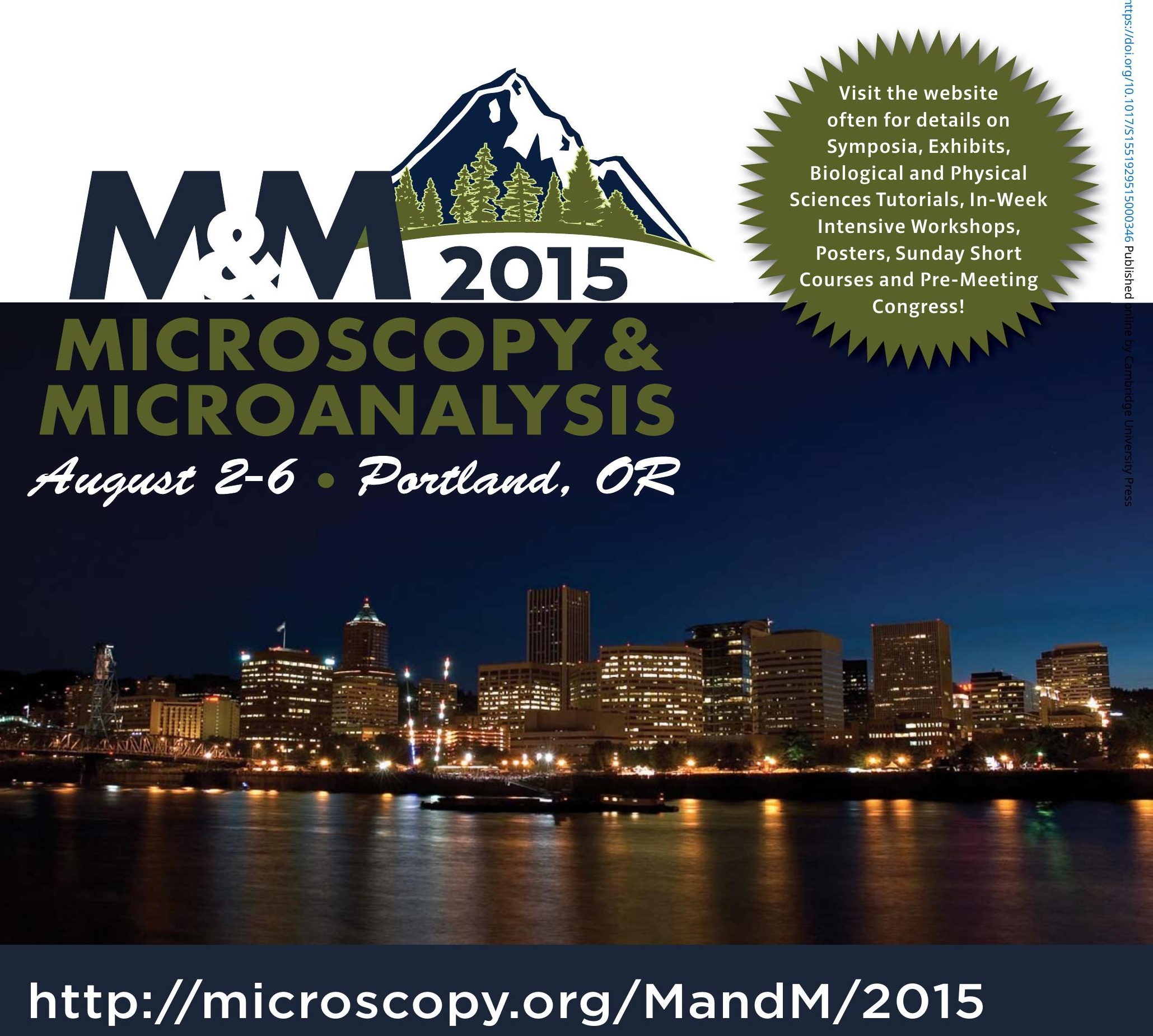

\section{http://microscopy.org/MandM/2015}
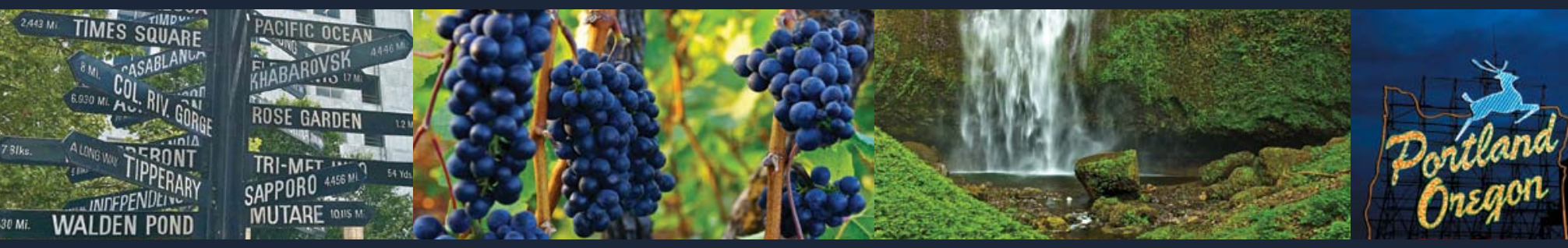

Program Information | Information for Students | Meeting Awards | Exhibitor List \& Floor Plan Online Registration (opens February 2015) | Portland Hotel Information (reservations available February 2015) 